\title{
High spatial entanglement via chirped quasi-phase-matched optical parametric down-conversion
}

\author{
Jiří Svozilík, ${ }^{1,2, *}$ Jan Peřina, Jr., ${ }^{2}$ and Juan P. Torres ${ }^{1,3}$ \\ ${ }^{1}$ ICFO_Institut de Ciencies Fotoniques, Mediterranean Technology Park, 08860, Castelldefels, Barcelona, Spain \\ ${ }^{2}$ Palacký University, RCPTM, Joint Laboratory of Optics, 17 listopadu 12, 77146 Olomouc, Czech Republic \\ ${ }^{3}$ Department of Signal Theory and Communications, Universitat Politecnica Catalunya, Campus Nord D3, 08034 Barcelona, Spain
}

(Received 22 August 2012; published 15 November 2012)

\begin{abstract}
By making use of the spatial shape of paired photons, parametric down-conversion allows the generation of two-photon entanglement in a multidimensional Hilbert space. How much entanglement can be generated in this way? In principle, the infinite-dimensional nature of the spatial degree of freedom renders unbounded the amount of entanglement available. However, in practice, the specific configuration used, namely, its geometry, the length of the nonlinear crystal, and the size of the pump beam, can severely limit the value that could be achieved. Here we show that the use of quasi-phase-matching engineering allows one to increase the amount of entanglement generated, reaching values of tens of ebits of entropy of entanglement under different conditions. Our work thus opens a way to fulfill the promise of generating massive spatial entanglement under a diverse variety of circumstances, some more favorable for its experimental implementation.
\end{abstract}

DOI: 10.1103/PhysRevA.86.052318 PACS number(s): 03.67.Bg, 42.50.Dv, 03.65.Aa, 42.65.Lm

\section{INTRODUCTION}

Entanglement is a genuine quantum correlation between two or more parties, with no analog in classical physics. During the last few decades it has been recognized as a fundamental tool in several quantum-information protocols, such as quantum teleportation [1], quantum cryptography [2], quantum key distribution [3], and distributed quantum computing [4].

Nowadays, spontaneous parametric down-conversion (SPDC), a process where the interaction of a strong pump beam with a nonlinear crystal mediates the emission of two lowerfrequency photons (signal and idler), is a very convenient way to generate photonic entanglement [5]. Photons generated in SPDC can exhibit entanglement in the polarization degree of freedom [6], frequency [7], and spatial shape [8,9]. One can also make use of a combination of several degrees of freedom [10,11].

Two-photon entanglement in the polarization degree of freedom is undoubtedly the most common type of generated entanglement, due to both its simplicity and that it suffices to demonstrate a myriad of important quantum-information applications. But the amount of entanglement is restricted to 1 ebit of entropy of entanglement [12], since each photon of the pair can be generally described by the superposition of two orthogonal polarizations (two-dimensional Hilbert space). On the other hand, frequency and spatial entanglement occurs in an infinite dimensional Hilbert space, offering thus the possibility to implement entanglement that inherently lives in a higher-dimensional Hilbert space (qudits).

The entangling of systems in higher-dimensional systems (frequency and spatial degrees of freedom) is important both for fundamental and applied reasons. For example, noise and decoherence tend to degrade quickly quantum correlations. However, theoretical investigations predict that physical systems with increasing dimensions can maintain nonclassical correlations in the presence of more hostile noise $[13,14]$.

*jiri.svozilik@icfo.es
Higher-dimensional states can also exhibit unique outstanding features. The potential of higher-dimensional quantum systems for practical applications is clearly illustrated in the demonstration of the so-called quantum coin tossing, where the power of higher-dimensional spaces is clearly visible [15].

The amount of spatial entanglement generated depends of the SPDC geometry used (collinear vs noncollinear), the length of the nonlinear crystal $(L)$, and the size of the pump beam $\left(w_{0}\right)$. To obtain an initial estimate, let us consider a collinear SPDC geometry. Under certain approximations [16], the entropy of entanglement can be calculated analytically. Its value can be shown to depend on the ratio $L / L_{d}$, where $L_{d}=k_{p} w_{0}^{2} / 2$ is the Rayleigh range of the pump beam and $k_{p}$ is its longitudinal wave number. Therefore, large values of the pump beam waist $w_{0}$ and short crystals are ingredients for generating high entanglement [17]. However, the use of shorter crystals also reduces the total flux-rate of generated entangled photon pairs. Moreover, certain applications might benefit from the use of focused pump beams. For instance, for an $L=1$-mm-long stoichiometric lithium tantalate (SLT) crystal, with pump beam waist $w_{0}=100 \mu \mathrm{m}$, pump wavelength $\lambda_{p}=400 \mathrm{~nm}$, and extraordinary refractive index $n_{e}\left(\lambda_{p}\right)=$ 2.27857 [18], one obtains $E \sim 8.5$ [16]. For a longer crystal of $L=20 \mathrm{~mm}$, the amount of entanglement is severely reduced to $E \sim 4.2$ ebits.

We put forward here a scheme to generate massive spatial entanglement, i.e., a staggering large value of the entropy of entanglement, independently of some relevant experimental parameters such as the crystal length or the pump beam waist. This would allow even larger amounts of entanglement than possible nowadays to be reached with the usual configurations used or this would allow the same amount of entanglement to be obtained but with other values of the nonlinear crystal length or the pump beam waist better suited for specific experiments.

Our approach is based on a scheme originally used to increase the bandwidth of parametric down-conversion [19-21]. A schematic view of the SPDC configuration is shown in Fig. 1. It makes use of chirped quasi-phase-matching (QPM) gratings with a linearly varying spatial frequency given by $K_{g}(z)=K_{0}-\alpha(z+L / 2)$, where $K_{0}$ is the grating's spatial 


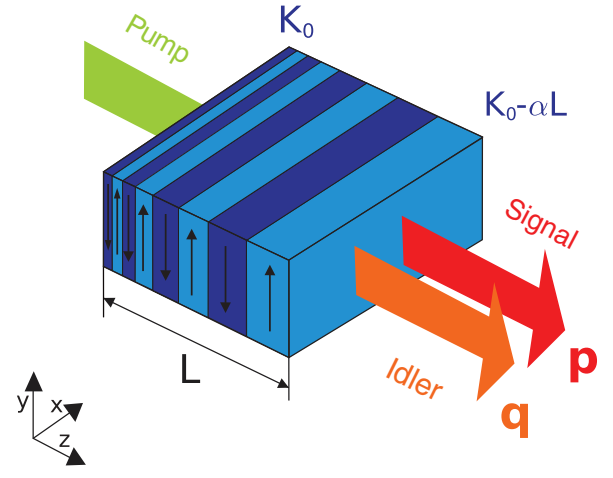

FIG. 1. (Color online) Scheme of SPDC in a linearly chirped quasi-phase-matched nonlinear crystal. The pump beam is a Gaussian beam, and $\mathbf{p}$ and $\mathbf{q}$ designate the transverse wave numbers of the signal and idler photons, respectively. $K_{0}$ is the grating wave vector at the input face of the nonlinear crystal, and $K_{0}-\alpha L$ is the grating wave vector at its output face. The signal and idler photons can have different polarizations or frequencies. The different colors (or different direction of arrows) represent domains with different signs of the nonlinear coefficient.

frequency at its entrance face $(z=-L / 2)$ and $\alpha$ is a parameter that represents the degree of linear chirp. The period of the grating at distance $z$ is $p(z)=2 \pi / K_{g}(z)$, so that the parameter $\alpha$ is written as

$$
\alpha=\frac{2 \pi}{L} \frac{p_{f}-p_{i}}{p_{f} p_{i}},
$$

where $p_{i}$ is the period at the entrance face of the crystal and $p_{f}$ is the period at its output face.

The key idea is that at different points along the nonlinear crystal, signal and idler photons with different frequencies and transverse wave numbers can be generated, since the continuous change of the period of the QPM gratings allows the fulfillment of the phase-matching conditions for different frequencies and transverse wave numbers. If appropriately designed narrow-band interference filters allow one to neglect the frequency degree of freedom of the two-photon state, then the linearly chirped QPM grating enhances only the number of spatial modes generated, leading to a corresponding enhancement of the amount of generated spatial entanglement.

\section{THEORETICAL MODEL}

In order to determine how much spatial entanglement can be generated in SPDC with the use of chirped QPM, let us consider a nonlinear optical crystal illuminated by a quasi-monochromatic laser Gaussian pump beam of waist $w_{0}$. Under conditions of collinear propagation of the pump, signal, and idler photons with no Poynting vector walk-off, which would be the case of a noncritical type II quasi-phasematched configuration, the amplitude of the quantum state of the generated two-photon pair generated in SPDC reads in transverse wave number space

$$
|\Psi\rangle=\int d \mathbf{p} d \mathbf{q} \Psi(\mathbf{p}, \mathbf{q})|\mathbf{p}\rangle_{s}|\mathbf{q}\rangle_{i},
$$

where $\mathbf{p}(\mathbf{q})$ is the transverse wave number of the signal (idler) photon. $\Psi$ is the joint amplitude of the two-photon state, so that $|\Psi(\mathbf{p}, \mathbf{q})|^{2}$ is the probability to detect a signal photon with transverse wave number $\mathbf{p}$ in coincidence with an idler photon with q.

The joint amplitude that describes the quantum state of the paired photons generated in a linearly chirped QPM crystal, using the paraxial approximation, is equal to

$$
\begin{aligned}
\Psi(\mathbf{p}, \mathbf{q})= & C \exp \left(-\frac{w_{0}^{2}}{4}|\mathbf{p}+\mathbf{q}|^{2}\right) \int_{-L / 2}^{L / 2} d z \\
& \times \exp \left[i \frac{|\mathbf{p}-\mathbf{q}|^{2}}{2 k_{p}} z+i \alpha\left(z+\frac{L}{2}\right) z\right],
\end{aligned}
$$

where $C$ is a normalization constant ensuring $\int d \mathbf{q} \int d \mathbf{p}|\Psi(\mathbf{p}, \mathbf{q})|^{2}=1$. Notice that the value of $K_{0}=2 \pi / p(-L / 2)$ does now show up in Eq. (3), since we make use of the fact that there is phase matching for $\mathbf{p}=\mathbf{q}=0$ at certain locations inside the nonlinear crystal, which in our case it turns out to be the input face $(z=-L / 2)$.

After integration along the $z$ axis one obtains

$$
\begin{aligned}
\Psi(\mathbf{p}, \mathbf{q})= & C \sqrt{\frac{i \pi}{4 \alpha}} \exp \left[-\frac{w_{0}^{2}}{4}|\mathbf{p}+\mathbf{q}|^{2}\right. \\
& \left.-i\left(\frac{\alpha L^{2}}{16}+\frac{L|\mathbf{p}-\mathbf{q}|^{2}}{8 k_{p}}+\frac{|\mathbf{p}-\mathbf{q}|^{4}}{16 \alpha k_{p}^{2}}\right)\right] \\
& \times\left[\operatorname{erf}\left(\frac{3 \sqrt{\alpha} L}{4 \sqrt{i}}+\frac{|\mathbf{p}-\mathbf{q}|^{2}}{4 k_{p} \sqrt{i \alpha}}\right)\right. \\
& \left.-\operatorname{erf}\left(-\frac{\sqrt{\alpha} L}{4 \sqrt{i}}+\frac{|\mathbf{p}-\mathbf{q}|^{2}}{4 k_{p} \sqrt{i \alpha}}\right)\right]
\end{aligned}
$$

where erf refers to the error function. Notice that Eq. (4) is similar to the one describing the joint spectrum of photon pairs in the frequency domain, when the spatial degree of freedom is omitted $[20,21]$. The reason is that both equations originate in phase-matching conditions along the propagation direction ( $z$ axis).

Since all the configuration parameters that define the down-conversion process show rotational symmetry along the propagation direction $z$, the joint amplitude given by Eq. (4) can be written as

$$
\Psi(\mathbf{p}, \mathbf{q})=\sum_{l=-\infty}^{\infty} B_{l}(p, q) e^{i l\left(\varphi_{p}-\varphi_{q}\right)} .
$$

Here, we have made use of polar coordinates in the transverse wave-vector domain for the signal photons, $\mathbf{p}=\left(p \cos \varphi_{p}, p \sin \varphi_{p}\right)$, and the idler photons, $\mathbf{q}=$ $\left(q \cos \varphi_{q}, q \sin \varphi_{q}\right)$, where $p$ and $q$ are the corresponding radial coordinates and $\varphi_{p, q}$ are the azimuthal angles. The specific dependence of the Schmidt decomposition on the azimuthal variables $\varphi_{p}$ and $\varphi_{p}$ reflects the conservation of the orbital angular momentum (OAM) in this SPDC configuration [22], so that a signal photon with OAM winding number $+l$ is always accompanied by a corresponding idler photon with OAM winding number $-l$. The probability of such coincidence detection for each value of $l$ is the spiral spectrum [23] of the two-photon state, i.e., the set of values $P_{l}=$ $\int p d p q d q\left|B_{l}(p, q)\right|^{2}$. Recently, the spiral spectra of some selected SPDC configurations have been measured [24]. 
The Schmidt decomposition $[25,26]$ of the spiral function, i.e., $B_{l}(p, q)=\sum_{n=0}^{\infty} \sqrt{\lambda_{n l}} f_{n l}(p) g_{n l}(q)$, is the tool to quantify the amount of entanglement present. $\lambda_{n l}$ are the Schmidt coefficients (eigenvalues), and the modes $f_{n l}$ and $g_{n l}$ are the Schmidt modes (eigenvectors). Here we obtain the Schmidt decomposition by means of a singular-value decomposition method. Once the Schmidt coefficients are obtained, one can obtain the entropy of entanglement as $E=-\sum_{n l} \lambda_{n l} \log _{2} \lambda_{n l}$. An estimation of the overall number of spatial modes generated is obtained via the Schmidt number $K=1 / \sum_{n l} \lambda_{n l}^{2}$, which can be interpreted as a measure of the effective dimensionality of the system. Finally, the spiral spectrum is obtained as $P_{l}=\sum_{n} \lambda_{n l}$.

\section{DISCUSSION}

For the sake of comparison, let us consider first the usual case of a QPM SLT crystal with no chirp, i.e., $\alpha=0 \mu \mathrm{m}^{-2}$, and length $L=20 \mathrm{~mm}$, pumped by a Gaussian beam with beam waist $w_{0}=100 \mu \mathrm{m}$ and wavelength $\lambda_{p}=400 \mathrm{~nm}$. In
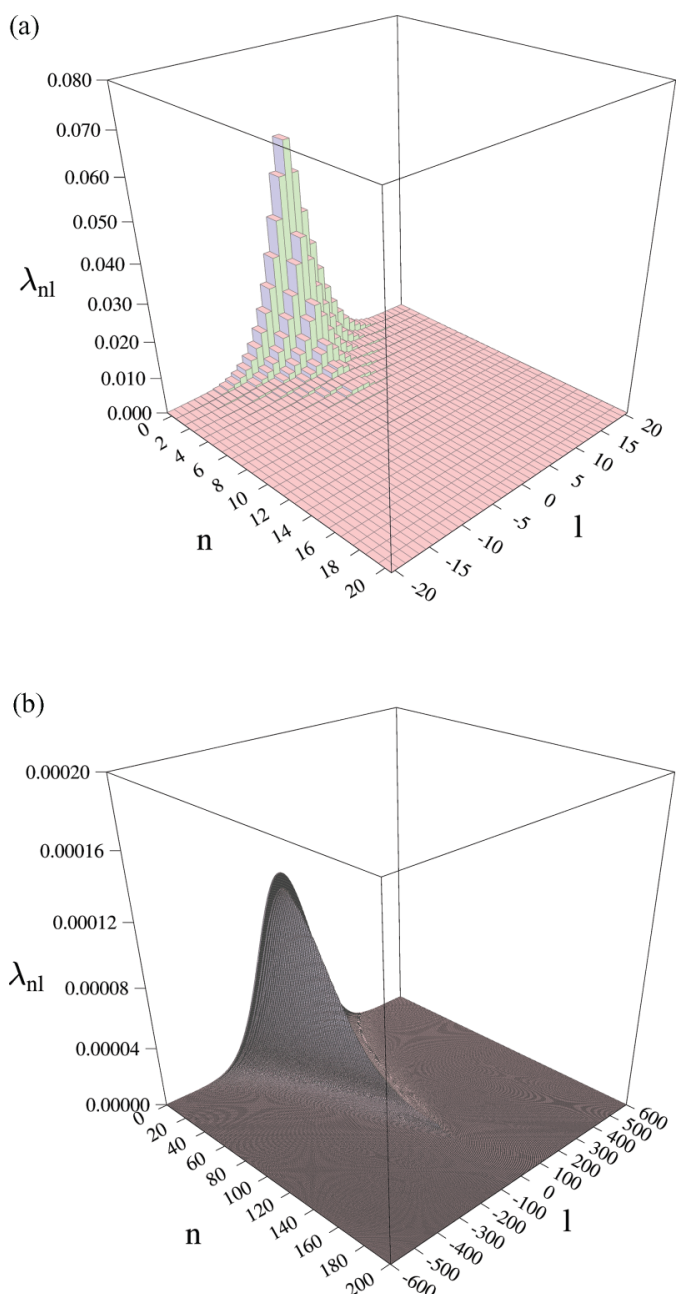

FIG. 2. (Color online) Weight of the Schmidt coefficients $\lambda_{n l}$ for (a) $\alpha=0 \mu \mathrm{m}^{-2}$ and (b) $\alpha=10 \times 10^{-6} \mu \mathrm{m}^{-2}$. The nonlinear crystal length is $L=20 \mathrm{~mm}$ and the pump beam waist is $w_{0}=100 \mu \mathrm{m}$. this case, the integration of Eq. (3) leads to [27]

$$
\Psi(\mathbf{p}, \mathbf{q})=C \exp \left(-\frac{w_{0}^{2}}{4}|\mathbf{p}+\mathbf{q}|^{2}\right) \operatorname{sinc}\left(\frac{L|\mathbf{p}-\mathbf{q}|^{2}}{4 k_{p}}\right) .
$$

The Schmidt coefficients are plotted in Fig. 2(a), and the corresponding spiral spectrum is shown in Fig. 3(a). The main contribution to the spiral spectrum comes from the spatial modes with $l=0$. The entropy of entanglement for this case is $E=6.4$ ebits and the Schmidt number is $K=42.9$.

Nonzero values of the chirp parameter $\alpha$ lead to an increase of the number of generated modes, as it can be readily seen in Fig. 2(b) for $\alpha=10 \times 10^{-6} \mu \mathrm{m}^{-2}$ and $w_{0}=100 \mu \mathrm{m}$. This broadening effect is also reflected in the corresponding broadening of the spiral spectrum, as shown in Fig. 3(b). Indeed, Fig. 4(a) shows that the entropy of entanglement increases with increasingly larger values of the chirping parameter, even though for a given value of $w_{0}$ its increase saturates for large values of $\alpha$. For $w_{0}=300 \mu \mathrm{m}$ and $\alpha=$ $10 \times 10^{-6} \mu \mathrm{m}^{-2}$, we reach a value of $E=16.6$ ebits. On the contrary, the Schmidt number $K$ rises linearly with $\alpha$, as can be observed in Fig. 4(b), for all values of $w_{0}$. For sufficiently large values of $w_{0}$ and $\alpha, K$ reaches values of several thousands of spatial modes, i.e., $K=87113$ for the same $w_{0}$ and $\alpha$. For large values of $E$, a further increase of $E$ requires an even much larger increase of the number of spatial modes involved, which explains why an increase of the number of modes involved only produces a modest increase of the entropy of entanglement. Notice that the spiral spectrum presented in Fig. 3(b) is discrete. Notwithstanding, it might
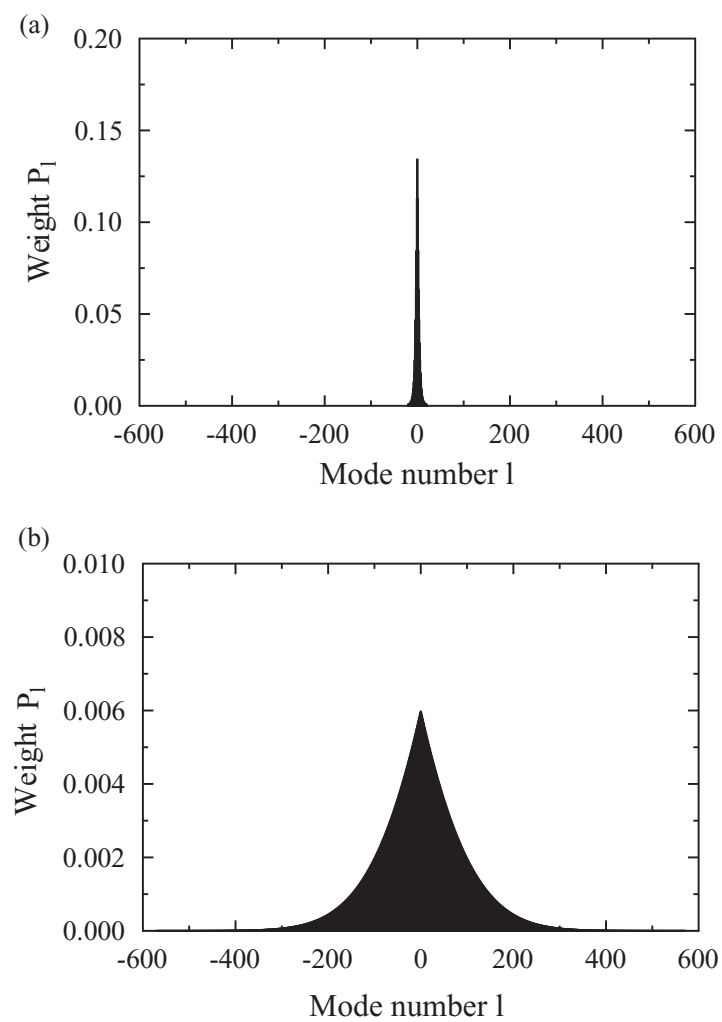

FIG. 3. The spiral spectrum $P_{l}$ for (a) $\alpha=0 \mu \mathrm{m}^{-2}$ and (b) $\alpha=$ $10 \times 10^{-6} \mu \mathrm{m}^{-2}$. The nonlinear crystal length is $L=20 \mathrm{~mm}$ and the pump beam waist is $w_{0}=100 \mu \mathrm{m}$. 
(a)

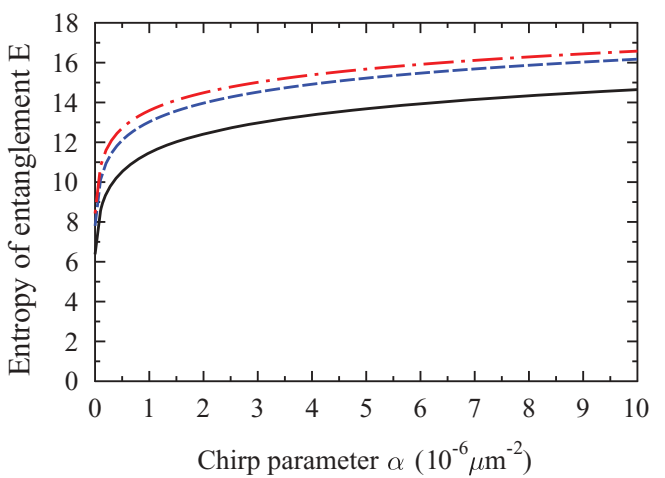

(b)

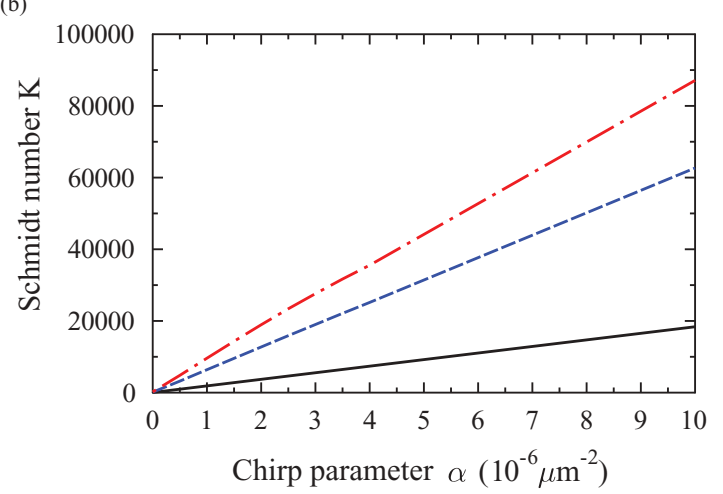

FIG. 4. (Color online) (a) The entropy of entanglement $E$ and (b) the Schmidt number $K$ as a function of the chirp parameter $\alpha$ for $w_{0}=100 \mu \mathrm{m}$ (solid black line), $w_{0}=200 \mu \mathrm{m}$ (dashed blue line), and $w_{0}=300 \mu \mathrm{m}$ (dotted-and-dashed red line).

look continuous since it is the result of the presence of several hundreds of OAM modes with slightly decreasing weights.

We have discussed entanglement in terms of transverse modes which arise from the Schmidt decomposition of the twophoton amplitude and, as such, they attain appreciable values in the whole transverse plane. Alternatively, the existing spatial correlations between the signal and idler photons can also be discussed using second-order intensity correlation functions [28]. In this approach, correlations are quantified by the size of the correlated area $(\Delta \mathbf{p})$ where it is highly probable to detect a signal photon provided that its idler twin has been detected with a fixed transverse wave vector $\mathbf{q}$. We note that the azimuthal width of the correlated area decreases with the increasing width of the distribution of Schmidt eigenvalues along the OAM winding number $l$. On the other hand, the increasing width of the distribution of Schmidt eigenvalues along the remaining number $n$ results in a narrower radial extension of the correlated area. An increase in the number of modes $K$ results in a diminishing correlation area, both in the radial and azimuthal directions. The correlated area drops to zero in the limit of plane-wave pumping, where it attains the form of a $\delta$ function. The use of such correlations in parallel processing of information represents the easiest way for the exploitation of the massively multimode character of the generated beams.

For the sake of comparison, when considering frequency entanglement, the entropy of entanglement depends on the ratio between the bandwidth of the pump beam (typically $\left.B_{p} \sim 5 \mathrm{MHz}\right)$ and the bandwidth of the down-converted two-photon state $\left(B_{\mathrm{dc}}\right)[29,30]$. For type II SPDC, one typically has values of $E \sim 1-2$ [7]. Increasing the bandwidth of the two-photon state, one can reach values of $B_{\mathrm{dc}}>1000 \mathrm{THz}$, therefore allowing typical ratios greater than $B_{\mathrm{dc}} / B_{p} \gg 10^{8}$, with $E>25$ [31].

\section{CONCLUSION}

In conclusion, we have presented a way to increase significantly the amount of two-photon spatial entanglement generated in SPDC by means of the use of chirped quasiphase-matching nonlinear crystals. This opens the door to the generation of high entanglement under various experimental conditions, such as different crystal lengths and sizes of the pump beam.

QPM engineering can also be an enabling tool to generate truly massive spatial entanglement, with state of the art QPM technologies [20] potentially allowing entropies of entanglement of tens of ebits to be reached. Therefore, the promise of reaching extremely high degrees of entanglement, offered by the use of the spatial degree of freedom, can be fulfilled with the scheme put forward here. The experimental tools required are available nowadays. The use of extremely high degrees of spatial entanglement, as consider here, would demand the implementation of high aperture optical systems. For instance, for a spatial bandwidth of $\Delta \mathbf{p} \sim 2 \mu \mathrm{m}^{-1}$, the aperture required for $\lambda_{p}=400 \mathrm{~nm}$ is $\Delta \theta \sim 4^{\circ}-6^{\circ}$.

The shaping of QPM gratings is commonly used in the area of nonlinear optics for multiple applications such as beam and pulse shaping, harmonic generation, and all-optical processing [32]. In the realm of quantum optics, its uses are not so widespread, even though QPM engineering has been considered, and experimentally demonstrated, as a tool for spatial $[33,34]$ and frequency [20] control of entangled photons. In view of the results obtained here concerning the enhancement of the degree of spatial entanglement, it could be possible to devise new types of gratings that turn out to be beneficial for other applications in the area of quantum optics.

\section{ACKNOWLEDGMENTS}

This work was supported by the Government of Spain (Project No. FIS2010-14831) and the European Union (Project No. PHORBITECH, FET-Open 255914). J.S. thanks Project No. FI-DGR 2011 of the Catalan Government. This work has also been supported in part by Projects No. COST OC 09026 and No. CZ.1.05/2.1.00/03.0058 of the Ministry of Education, Youth and Sports of the Czech Republic and by Project No. PrF-2012-003 of Palacký University.
[1] C. H. Bennett, G. Brassard, C. Crépeau, R. Jozsa, A. Peres, and W. K. Wootters, Phys. Rev. Lett. 70, 1895 (1993).

[2] A. K. Ekert, Phys. Rev. Lett. 67, 661 (1991).
[3] G. Ribordy, J. Brendel, J. D. Gautier, N. Gisin, and H. Zbinden, Phys. Rev. A 63, 012309 (2000).

[4] A. Serafini, S. Mancini, and S. Bose, Phys. Rev. Lett. 96, 010503 (2006). 
[5] J. P. Torres, K. Banaszek, and I. A. Walmsley, Prog. Opt. 56, 227 (2011).

[6] P. G. Kwiat, K. Mattle, H. Weinfurter, A. Zeilinger, A. V. Sergienko, and Y. Shih, Phys. Rev. Lett. 75, 4337 (1995).

[7] C. K. Law, I. A. Walmsley, and J. H. Eberly, Phys. Rev. Lett. 84, 5304 (2000).

[8] H. H. Arnaut and G. A. Barbosa, Phys. Rev. Lett. 85, 286 (2000).

[9] A. Mair, A. Vaziri, G. Weihs, and A. Zeilinger, Nature (London) 412, 313 (2001).

[10] J. T. Barreiro, N. K. Langford, N. A. Peters, and P. G. Kwiat, Phys. Rev. Lett. 95, 260501 (2005).

[11] E. Nagali, F. Sciarrino, F. De Martini, L. Marrucci, B. Piccirillo, E. Karimi, and E. Santamato, Phys. Rev. Lett. 103, 013601 (2009).

[12] For a two-photon state with density matrix $\rho_{12}$, the entropy of entanglement is defined as $E=-\operatorname{Tr}\left(\rho_{1} \log _{2} \rho_{1}\right)$, where $\rho_{1}=$ $\operatorname{Tr}_{2} \rho_{12}$ is the partial trace over the variables describing subsystem 2 of the global density matrix. The entropy of entanglement of a maximally entangled quantum state, whose two parties live in a $d$-dimensional system, is $\log _{2} d$. Since the state of polarization of a single photon is a two-dimensional system, the maximum entropy of entanglement is 1 .

[13] D. Kaszlikowski, P. Gnacinski, M. Zukowski, W. Miklaszewski, and A. Zeilinger, Phys. Rev. Lett. 85, 4418 (2000).

[14] D. Collins, N. Gisin, N. Linden, S. Massar, and S. Popescu, Phys. Rev. Lett. 88, 040404 (2002).

[15] G. Molina-Terriza, A. Vaziri, R. Ursin, and A. Zeilinger, Phys. Rev. Lett. 94, 040501 (2005).

[16] The approximation consists of substituting the sinc function appearing in Eq. (6) by a Gaussian function, i.e., sinc $b x^{2} \approx$ $\exp \left[-\gamma b x^{2}\right]$, with $\gamma=0.499$, so that both functions coincide at the $1 / 2$ intensity. For a detailed calculation, see K. W. Chan, J. P. Torres, and J. H. Eberly, Phys. Rev. A 75, 050101(R) (2007).
[17] S. S. R. Oemrawsingh, X. Ma, D. Voigt, A. Aiello, E. R. Eliel, G. W. 't Hooft, and J. P. Woerdman, Phys. Rev. Lett. 95, 240501 (2005).

[18] A. Bruner, D. Eger, M. B. Oron, P. Blau, M. Katz, and S. Ruschin, Opt. Lett. 28, 194 (2003).

[19] S. Carrasco, J. P. Torres, L. Torner, A. Sergienko, B. E. A. Saleh, and M. C. Teich, Opt. Lett. 29, 2429 (2004).

[20] M. B. Nasr, S. Carrasco, B. E. A. Saleh, A. V. Sergienko, M. C. Teich, J. P. Torres, L. Torner, D. S. Hum, and M. M. Fejer, Phys. Rev. Lett. 100, 183601 (2008).

[21] J. Svozilík and J. Peřina, Jr., Phys. Rev. A 80, 023819 (2009).

[22] C. I. Osorio, G. Molina-Terriza, and J. P. Torres, Phys. Rev. A 77, 015810 (2008).

[23] J. P. Torres, A. Alexandrescu, and L. Torner, Phys. Rev. A 68 , 050301 (2003).

[24] H. Di Lorenzo Pires, H. C. B. Florijn, and M. P. van Exter, Phys. Rev. Lett. 104, 020505 (2010).

[25] A. Ekert and P. L. Knight, Am. J. Phys. 63, 415 (1995).

[26] C. K. Law and J. H. Eberly, Phys. Rev. Lett. 92, 127903 (2004).

[27] S. P. Walborn, A. N. de Oliveira, S. Padua, and C. H. Monken, Phys. Rev. Lett. 90, 143601 (2003).

[28] M. Hamar, J. Peřina, Jr., O. Haderka, and V. Michálek, Phys. Rev. A 81, 043827 (2010).

[29] S. Parker, S. Bose, and M. B. Plenio, Phys. Rev. A 61, 032305 (2000).

[30] J. Peřina, Jr., Phys. Rev. A 77, 013803 (2008).

[31] M. Hendrych, X. Shi, A. Valencia, and J. P. Torres, Phys. Rev. A 79, 023817 (2009).

[32] D. S. Hum and M. M. Fejer, C. R. Phys. 8, 180 (2007).

[33] J. P. Torres, A. Alexandrescu, S. Carrasco, and L. Torner, Opt. Lett. 29, 376 (2004).

[34] X. Q. Yu, P. Xu, Z. D. Xie, J. F. Wang, H. Y. Leng, J. S. Zhao, S. N. Zhu, and N. B. Ming, Phys. Rev. Lett. 101, 233601 (2008). 